\title{
Vocal fold augmentation under local anaesthesia using autologous fascia
}

Kinnari, TJ

2018-06

Kinnari , TJ , Pietarinen , P \& Geneid , A 2018 , ' Vocal fold augmentation under local anaesthesia using autologous fascia ' , Clinical Otolaryngology , vol. 43 , no. 3 , pp. 989-991 . https://doi.org/10.1111/coa.13030

http://hdl.handle.net/10138/302421

https://doi.org/10.1111/coa.13030

publishedVersion

Downloaded from Helda, University of Helsinki institutional repository.

This is an electronic reprint of the original article.

This reprint may differ from the original in pagination and typographic detail.

Please cite the original version. 


\title{
Vocal fold augmentation under local anaesthesia using autologous fascia
}

\author{
T. J. Kinnari | P. Pietarinen | A. Geneid \\ Department of Otorhinolaryngology, Head and Neck Surgery, Helsinki University Hospital and University of Helsinki, Helsinki, Finland \\ Correspondence \\ T. Kinnari, Department of Otorhinolaryngology, Head and Neck Surgery, Helsinki University Hospital and University of Helsinki, Helsinki, Finland. \\ Email: teemu.j.kinnari@helsinki.fi
}

\section{1 | INTRODUCTION}

Vocal fold augmentation by injection of autologous fascia has been used since the 1990s. As an autologous implant material, fascia is safe from risks arising from allergic or other foreign body adverse reactions. Fascia injection has an excellent safety record in the 500 operations carried out in the Helsinki University ENT clinic throughout the last two decades. ${ }^{1}$ There are numerous articles showing the excellent durability of fascia as an augmentation material. It has been shown that free fascia grafts survive as well-organised living fibrous tissue with a vascular supply providing a long-term augmentation of the atrophied paralysed vocal fold without disturbing its subepithelial layer. ${ }^{1}$ Furthermore, fascia has been used to provide better closure in cases of a sulcus vocalis. ${ }^{2}$

The augmentation result remains stable 3-10 years after the fascia injection regardless of the length of follow-up, the delay from paralysis to surgery or the age of the patient. ${ }^{3}$ Furthermore, the short-term resorption rate of autologous fascia is low, which reduces the need for overcorrection compared to other augmentation materials such as autologous fat or synthetic materials such as calcium hydroxylapatite ( $\mathrm{CaHA}$ ) that are resorbable within one to a few years. ${ }^{4}$ The fascia injection is only possible through a large gauge needle. The procedure is normally performed under general anaesthesia using jet ventilation. We use the Microfrance MCL55 pressure injector and a Karl Storz 27200S 19-gauge straight needle. $^{5}$ A rebirth of office-based vocal fold injections has occurred during the last decade, influenced by new high-quality endoscopic techniques and the introduction of new, thin materials that can be injected through a small gauge needle. Procedures under local anaesthesia make it possible to cater to patients unfit for general anaesthesia, providing aid to fragile and weak persons with vocal fold paralysis. However, the majority of the synthetic materials in use have short- or medium-term effects, and the resorption of these materials creates a need for both substantial overcorrection and reinjection within 6-24 months. To avoid several re-operations in patients unfit for anaesthesia, we have started vocal fold augmentations under local anaesthesia using autologous fascia. In this article and attached video, we introduce, step-by-step, how the procedure is performed.

\section{TECHNICAL DESCRIPTION}

Ethical consideration: informed consent was given by the patients who underwent fascia injection under local anaesthesia. Vocal fold injection with fascia is a routine procedure in Helsinki University Hospital with no need for ethical approval.

As a large gauge needle is needed for injection of the fascia, we decided on a per-oral route for the operation. The procedure is performed with a Karl Storz 27200S multi-use needle (Karl Storz GmbH, Tuttlingen, Germany), originally made for Teflon injections, which has been curved by an experienced technician for our purpose (Figure 1). The standard MicroFrance MCL55 injector (Integra MicroFrance SAS, Saint Aubin le Monial, France) is used because the fascia needs to be injected with high pressure.

For the fascia harvesting, the patient is in a supine position. A piece of fascia lata is collected from the anterolateral area of the thigh, halfway between the knee and hip joint, after administration of $1 \%$ lidocaine with $1 / 10000$ adrenaline for the skin anaesthesia. The skin and subcutaneous fat are elevated to expose the fascia. A roughly circular fascia slice of $4-5 \mathrm{~cm}$ in diameter is sharply dissected from the surface of the muscle, and an amount of up to $2 \mathrm{~mL}$ of fat is collected as well. Two subcutaneous stitches are usually enough to close the wound without the need to suture the fascia layer. The fascia sample is minced on a glass plate using scissors until it becomes the consistency of toothpaste. It is advisable to occasionally dip the scissor tip in saline to keep the minced fascia moist. After careful mincing, the fascia is introduced to the injector. After the fascia is loaded, 1-2 $\mathrm{mL}$ of fat tissue is added to the injector as well to serve as a vehicle to ensure that all fascia can be injected out of the long needle. 


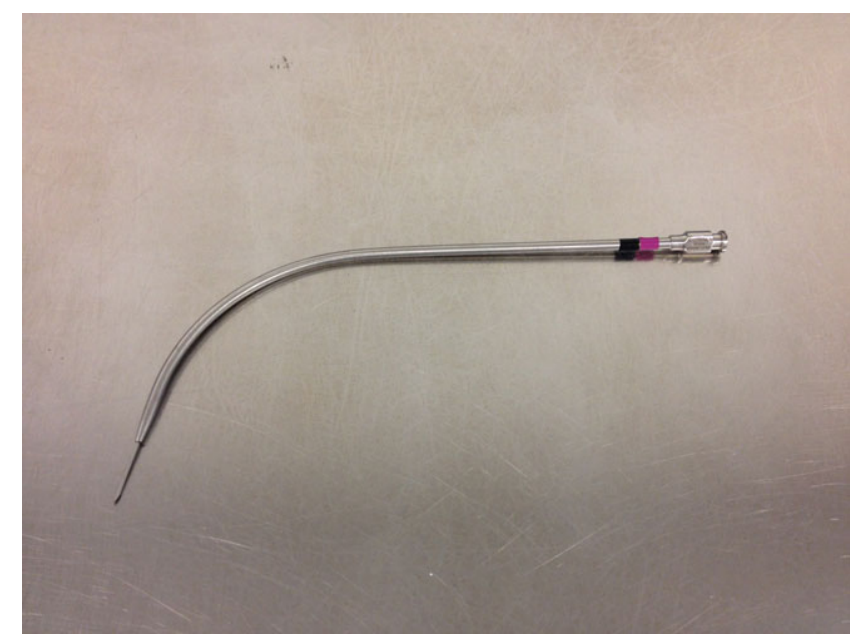

FIGURE 1 Curved needle used for injections

The injection is performed in a seated position under nasofiberoscopic view (Figure 2). For this purpose, the patient's nose is anaesthetised using $1 \%$ lidocaine spray or cotton stick. With the endoscopic view, $2-4 \mathrm{~mL}$ of $4 \%$ lidocaine is administered per orally to the hypopharynx and larynx using a curved cannula. The curved suction cannula used in sinus surgery is well-suited for this purpose. The patient is asked to hold their breath during the administration of the anaesthetic and then phonate "ee" so that the anaesthetic spreads all over the glottis and supraglottic space. Within minutes, the larynx is completely numb. The needle then is introduced to the larynx through the patient's mouth, and the fascia is injected at 1-2 points in the posterolateral part of the thyroarytenoid muscle. The need for overcorrection is minimal. The patient's voice can be tested instantly, and fine-tuning can be carried out by inserting more fascia if needed. Two days of voice rest after the injection is recommended to avoid vocal muscle strain pushing the fascia out through the insertion point. The operation can be carried out in facilities prepared for minor operative

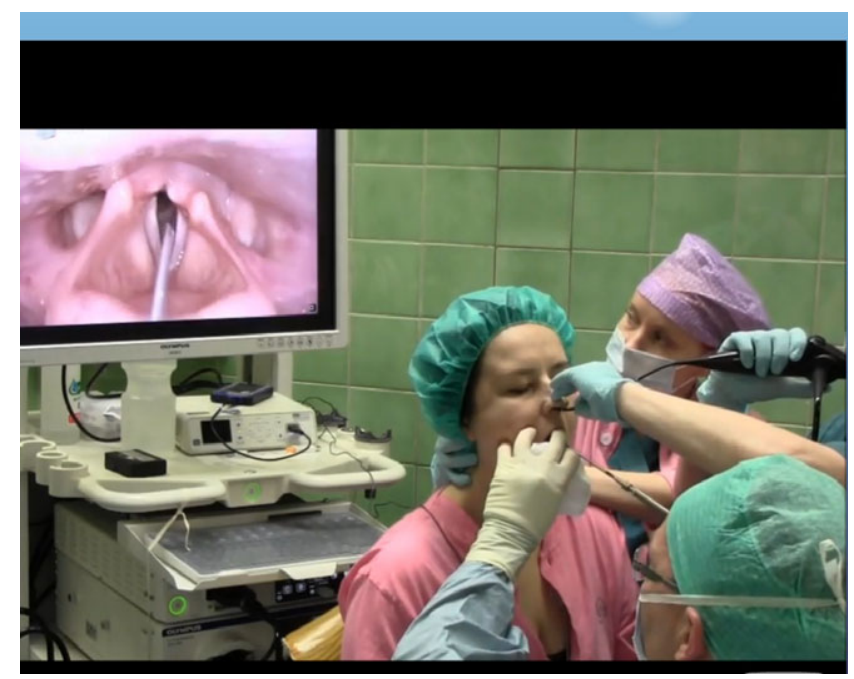

FIGURE 2 Set-up for injection of fascia under local anaesthesia

\section{Keypoints}

- Vocal fold augmentation by injection of autologous fascia has been used since the 1990s with excellent safety record in 500 operations carried out under general anaesthesia. It is safe from risks arising from allergic or other foreign body adverse reactions. Fascia provides long-term augmentation and remains stable 3-10 years after injection.

- Vocal fold augmentation under local anaesthesia is excellent alternative for those unfit for general anaesthesia, providing aid to fragile and weak persons with vocal fold paralysis. However, all of available synthetic materials resorb within 6-24 months.

- Fascia injection under local anaesthesia provides the possibility for a long-term vocal fold medialisation while avoiding risk of general anaesthesia. Fascia lata harvesting and processing is easily carried out (as shown in video) followed by injection into the vocal fold. The whole procedure is performed under local anaesthesia. Procedure offers long-term vocal fold medialisation, fine-tuning the amount of the medialisation by immediate feedback from the patient's voice. Operation time is one hour.

procedures without an anaesthesiologist. A video of the operation is available with this article.

\section{DISCUSSION}

The rebirth of injection laryngoplasty under local anaesthesia arose from the ease of the procedure, as well as the ability to avoid the risks of general anaesthesia. However, with many of the currently available materials, the need for re-injection has been quite evident.

The injection of autologous fascia provides the possibility for a long-term vocal fold medialisation. Furthermore, it also allows for fine-tuning the amount of the medialisation by immediate feedback from the patient's voice.

The cost of using an operating theatre is markedly reduced under local anaesthesia by saving the time for induction and termination of general anaesthesia as well as recovery time. The procedure, being performed under local anaesthesia with an approximate duration of one hour, also protects the patient from the risks associated with general anaesthesia. Furthermore, the past 10 years have not shown any complications accompanied with the harvesting of fascia lata at the donor site. Another benefit of the procedure is that injection with autologous fascia reduces the costs of artificial materials and repeated injections.

One could argue that injection of the vocal folds with autologous fascia is not widely used. We believe that the reason for this lies in the 
fact that the method used for processing harvested fascia is not widely known or seen. The video accompanying this article shows, among other details, how effortless the harvesting and mincing of fascia is.

In this manuscript, we present a new technique for vocal fold injection under local anaesthesia using autologous fascia. With the harvesting of the fascia, as well as its injection, under local anaesthesia, this procedure offers a way to reduce the risks associated with general anaesthesia. It also offers the possibility of instant feedback and fine-tuning the amount of fascia required based on the changes of the voice.

\section{CONFLICT OF INTEREST}

The authors do not have any financial or personal relationships that could influence this work. The authors have not received any financial support concerning this manuscript.

\section{ORCID}

A. Geneid ID http://orcid.org/0000-0003-1617-1181

\section{REFERENCES}

1. Reijonen P, Leivo I, Nevalainen T, Rihkanen H. Histology of injected autologous fascia in the paralyzed canine vocal fold. Laryngoscope. 2001;6:1068-1074.

2. Tsunoda K, Takanosawa M, Niimi S. Autologous transplantation of fascia into the vocal fold: a new phonosurgical technique for glottal incompetence. Laryngoscope. 1999;3:504-508.

3. Reijonen P, Tervonen H, Harinen K, Rihkanen H, Aaltonen L. Longterm results of autologous fascia in unilateral vocal fold paralysis. Eur Arch Otorhinolaryngol. 2009;8:1273-1278.

4. Mallur PS, Rosen CA. Vocal fold injection: review of indications, techniques, and materials for augmentation. Clin Exp Otorhinolaryngol. 2010;4:177-182.

5. Rihkanen $\mathrm{H}$. Vocal fold augmentation by injection of autologous fascia. Laryngoscope. 1998;1:51-54.

How to cite this article: Kinnari TJ, Pietarinen P, Geneid A. Vocal fold augmentation under local anaesthesia using autologous fascia. Clin Otolaryngol. 2018;43:989-991. https://doi.org/10.1111/coa.13030 